\title{
Dynamic Selection of Optimal Sub-portfolio - Application in the Regional Stock Exchange of Securities in West Africa (BRVM)
}

\author{
Seydina I. Dione ${ }^{1}$, Salimata G. Diagne ${ }^{1}$, Déthié Dione ${ }^{1}$, Youssou Gningue ${ }^{2}$ \\ ${ }^{1}$ Cheikh Anta Diop University, Dakar, Senegal \\ ${ }^{2}$ Laurentian University, Canada \\ Correspondence: Seydina I. Dione, Cheikh Anta Diop University, Dakar, Senegal. Tel: 221-77-525-73-57. E-mail: \\ seydinaissa.dione@ucad.edu.sn
}

Received: July 1, 2015 Accepted: July 22, 2015 Online Published: August 14, 2015

doi:10.5539/jmr.v7n3p198～URL: http://dx.doi.org/10.5539/jmr.v7n3p198

\begin{abstract}
In this paper, we consider a generalization of the mean-variance model of Markowitz, including a new limiting constraint on marginal returns through the PER (price earnings ratio), that we apply to an optimal portfolio selection in the Regional Stock Exchange of Securities in West africa (BRVM). We study the PER restriction impact through a dynamic selection of optimal sub-portfolios from an initial portfolio. We trace out dynamically the efficient frontiers of the sub-portfolios for different integer values of the PER. The main objective is to provide, for every level of performance (in term of returns) and for a fixed PER, the selection of optimal sub-portfolios that should lead, for a minimal risk, to the same performance as the original portfolio. We show that based on the PER, we can know for each level of performance, the securities to turn down to be able to define the best selection that converge to the optimal portfolio. Moreover, this work is a contribution to the development efforts of our financial systems and our West African stock market environment. The plots of the efficient frontiers of the sub-portfolios were carried out throughout data collected from the BRVM (Regional Stock Exchange Securities) in the period from October 2014 to February 2015.
\end{abstract}

Keywords: Mean-variance model, Minimal risk, Sub-portfolio, Price earnings ratio, Efficient frontiers convergence

\section{Introduction}

The main idea in a portfolio selection problem is to find out the very best way (optimal) to invest a defined capital among many financial stocks. What we name portfolio is given by each one of the multitude of possible affectations, through a linear combination, of this capital between those stocks. Markowitz (1952) was the first to introduce an approch (mean-variance model) to solve this kind of portfolio optimization problem of financial securities by posing the variance of returns as a measure of risk. The efficient frontier gives the set of the portfolios with a minimum risk for a given level of performance and then provides the optimum for any desired level of performance. Several critics of this approach were proved including the quadratic nature of the objective function and the calculation of the variance-covariance matrix. Thus to simplify the difficulties associated with the structure of Markowitz model, several alternative models have been proposed. Some authors such as Sharpe (1967), (1971) and Stone (1973) tried to linearize the portfolio choice problem. Rudd \& Rosenbeg (1979) showed that the Markowitz model in its classic formulation may not be able to satisfy a professional investor and propose an even more realistic portfolio management. More recently, several linear programming models have been developed for portfolio selection. Konno \& Yamazaki (1991), Zenios \& Kang (1993) and Speranza (1993) proposed the calculation of portfolio risk through the use of the mean absolute deviation. They also proposed an approach including asymmetric risk criterion which eliminates many problems related to this optimization model. With the development of linear models Yoshimoto (1996) has developed optimization system based on the mean-variance criterion. Hamza \& Janssen (1995) generalized standard models retaining the risk-return couple as a basic idea and suggesting a new risk measure defined as a convex combination of two semi-variances. They also proposed a general linear programming model based on separable programming techniques. Recently, a new measure of risk called Value at Risk (VaR) has been introduced in order to estimate the maximum loss that might occur at a quite probability during a given period. There are many models to assess the VaR, but its choice depends on the kind of portfolio and the datas used to estimate the parameters, Beder (1995), Jorion (2000). The different models that we present in this paper consist either in the maximization of the return for a given risk or in the minimization 
of this risk for a given return. More recently, El Hachloufi \& al. (2012) have suggested a method based on the classification and genetic algorithm in order to optimize a portfolio while acting simultaneously on the risk and the return. However, none of these imposes any parameter to guide the choice of individual shares that must constitute the portfolio.

On the other side, Fieldsend \& al. (2004) have studied the introduction of cardinal constraints in the portfolio optimization. Gilli \& al. (2000) have elaborated a synthesis of heuristic approachs in the optimal portfolio selection, whereas Schaerf (2002), Jobst \& al. (2001) are interested in the simulation as an option, particularly in face of discrete constraints about assets choice. Despite the outstanding progress in the financial modelling, as above-mentioned in the literature since the advent of Markowitz model, we are going to use this last model expectation-variance as well as the hypothesis in a more specific African stock exchange context. In addition, we are going to insert a constraint linked to the Price Earnings Ratio, by fixing a minimum threshold for each return of the assets that might constitute the portfolio. This constraint, which we want to study the impact, is very useful in the practice (Basu, 1977; Penman, 1996). This constraint should lead us, from an initial portfolio, to elaborate sub-portfolios composed of fewer assets and providing the same level of return as the initial portfolio for a minimum risk. So the Markowitz model was generalized including this constraint which turns it into a dynamic selection problem of sub-optimal portfolios, for dynamic values of the price earnings ratio. We focus on digital simulation appearance through the efficient frontier plotting of sub-portfolios from an initial portfolio of nineteen shares with the best PER in the first compartment of the Regional Stock Exchange of Securities in West Africa at the end of last quarter 2014. The results obtained with the sub-portfolios were compared with the efficient frontier of the initial portfolio to draw up for each performance level, the selection of sub-portfolio that should lead, at a minimal risk, to the same performance as the original portfolio and implicitly the securities for which no amount will be invested in for any risk level.

This paper is organized as follows: Section 1 is the introduction, section 2 presents the elements of portfolio theory and the new introduced notion called convergence of efficient frontiers. The mathematical formulation of the dynamic selection model of sub-portfolios is described in Section 3. In Section 4 we present some numerical experiments and finally in Section 5 we describe the experimental results.

\section{Portfolio Theory Elements}

\subsection{Return, PER and Sub-portfolio}

The return obtained at the time $t$ through an investment in a share is given by the difference between its course at the time $t$ plus dividends received over the period $[t-1, t]$ and its course at the time $t-1$ divided by the price at the time $t-1$ :

$$
r_{t}=\frac{\left(c_{t}+d_{t}\right)-c_{t-1}}{c_{t-1}}
$$

Wherein :

$c_{t}$ : Course of share $i$ at the end of period $t$.

$d_{t}$ : Dividends received at the end of the period.

The PER (price earnings ratio) of a share measures the number of years it takes to recover the invested amount in the event that this gain is constant. It evaluates thereby the dearness of a security relative to the prices of the corporate securities in the same sector (the higher the PER, the lower the action is considered cheap). It can be interpreted as an inverted yield according to Beaver \& al. (1978) and Penman (1996).

$$
p_{i}=\frac{1}{r_{i t}}
$$

wherein :

$$
\begin{aligned}
& p_{i}: \text { PER of the share } i \\
& r_{i t} \text { : Return of the share } i \text { at the time } t .
\end{aligned}
$$

However actual performance may differ from those obtained after the study of the PER for example because of a company growth policy for which the company in question chooses to reinvest instead of a total distribution of the 
result by way of dividend. In practice, $p_{i}$ is usually adjusted or round up so that we have $p_{i} \in \mathbb{N}^{*}$ to better facilitate its supervision.

The expected return of a share for the period $T$ is :

$$
\bar{r}_{i}=\frac{1}{T} \sum_{t=1}^{T} r_{i t}
$$

According to (Markowitz, 1952), the expected return $\bar{R}_{p}$ of a portfolio composed by $n$ shares having $\bar{r}_{i}$ as expected return is:

$$
\bar{R}_{p}=\sum_{i=1}^{n} \mathbf{z}_{i} \bar{r}_{i}
$$

wherein $\mathbf{z}_{1}, \ldots, \mathbf{z}_{n}$ are the portions of capital that the investor places respectively in the assets $i,(i=1, \ldots, n)$.

We will call sub-portfolio a portfolio of $k$ assets constituted from an initial portfolio of $n$ assets $(k \leq n)$.

\subsection{Portfolio Risk}

The risk of a financial asset is the uncertainty about the value of this asset at a future date. The variance, the mean absolute deviation, the semi-variance, the VaR (Value at Risk) and the CVaR (Conditional Value at Risk) are tools used to measure the risk.

The risk of a portfolio can be measured by one of the above-mentioned elements. It depends on three factors, namely:

- The risk of each security included in the portfolio,

- The degree of independence of changes in equity,

- The number of shares which compose the portfolio.

Suppose the variance as a measure of risk, Markowitz (1952) establishes the following expression for a portfolio of $n$ shares.

$$
\sigma^{2}\left(\bar{R}_{p}\right)=\sum_{i=1}^{n} \mathbf{z}_{i}^{2} \sigma^{2}\left(\bar{r}_{i}\right)+2 \sum_{i \neq j} \mathbf{z}_{i} \mathbf{z}_{j} \operatorname{cov}\left(\bar{r}_{i}, \bar{r}_{j}\right)
$$

\subsection{Efficient Frontier - Convergence of Efficient Frontiers}

\subsubsection{Efficient Frontier}

In an orthonormal axis system where we represent the risk in abscissa and the return in ordinate, the portfolio manager's objective is to maximize his return for a given risk or conversely to minimize risk for a given yield. Any portfolio is defined by the data of the torque corresponding risk-return and the efficient frontier is the representation of this set of optimal risk-return pairs (efficient) or efficient portfolios. We introduce the concepts of convergent efficient frontiers and those equivalent.

\subsubsection{Convergence and Equivalence of Efficient Frontiers}

Given two sets of portfolios composed from respectively $m$ and $n$ assets of a market $(m, n \in \mathbb{N}, m \neq n)$, let $f_{m}$ and $f_{n}$ be the efficient frontiers respectively associated with each of these compositions. Let $P_{m}$ (respectively $P_{n}$ ) be a portfolio made from the $m$ (respectively $n$ ) assets.

Definition $1 f_{m}$ and $f_{n}$ converge to the same value if from a certain level of risk, $f_{m}$ coincides with $f_{n}$, ie there is $\sigma_{0}$ strictly positive, such as at a same level of higher risk than $\sigma_{0}$, the optimum yields corresponding to each of the two different compositions of portfolio tend toward the same value.

$$
\begin{array}{r}
\exists \sigma_{0}>0, \forall\left(\sigma\left(P_{m}\right), \bar{R}\left(P_{m}\right)\right) \in f_{m},\left(\sigma\left(P_{n}\right), \bar{R}\left(P_{n}\right)\right) \in f_{n}, \quad \sigma\left(P_{m}\right) \geq \sigma_{0}, \sigma\left(P_{n}\right) \geq \sigma_{0} \\
\text { thus } \quad \sigma\left(P_{m}\right)=\sigma\left(P_{n}\right) \Longleftrightarrow \bar{R}\left(P_{m}\right)=\bar{R}\left(P_{n}\right)
\end{array}
$$

Definition $2 f_{m}$ is equivalent to $f_{n}$ if $f_{m}$ and $f_{n}$ converge to the same value at any level of risk where efficient portfolios are defined, ie at the same level of risk, the optimum yields for each of the two different compositions of 
portfolio tend toward the same value.

$$
\begin{array}{r}
\forall\left(\sigma\left(P_{m}\right), \bar{R}\left(P_{m}\right)\right) \in f_{m},\left(\sigma\left(P_{n}\right), \bar{R}\left(P_{n}\right)\right) \in f_{n}, \\
\sigma\left(P_{m}\right)=\sigma\left(P_{n}\right) \Longleftrightarrow \bar{R}\left(P_{m}\right)=\bar{R}\left(P_{n}\right)
\end{array}
$$

\section{Mathematical Formulation of the Problem}

We define our sub-portfolio optimization problem as following for $k<n$ ( $n$ total number of assets in the market ):

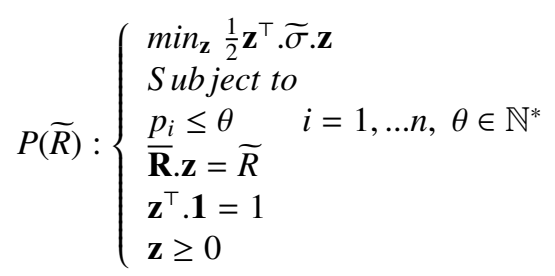

with :

- $p_{i}$ is the PER of the asset $i$

- $\theta$ the number of required years

- $k$ is the number of assets which compose the sub-portfolio, ie the number of assets such as $p_{i} \leq \theta$.

- $\widetilde{\sigma}$ is the covariance matrix of the $k$ assets composing the sub-portfolio,

- $\mathbf{z}$ is the column vector of portions of equity invested in the sub-portfolio,

- $\mathbf{1}$ is the column vector whose components are equal to 1 ,

- $\widetilde{R}$ is the required return,

- $\overline{\mathbf{R}}$ is the row vector of expected returns: $\overline{\mathbf{R}}=\left[\begin{array}{lllll}\bar{r}_{1} & \ldots & \bar{r}_{i} & \ldots & \bar{r}_{k}\end{array}\right]$.

Solve this problem would require to work with $(k+2)$ Lagrange multipliers and the Lagrangian function associated with the problem $P(\widetilde{R})$ can be written as following (Cornuejols G. et al. (2007))

$$
\mathfrak{f}\left(\mathbf{z}, \lambda, \mu ; \alpha_{i}, \ldots, \alpha_{k}\right)=\frac{1}{2} \mathbf{z}^{\top} \cdot \widetilde{\sigma} \cdot \mathbf{z}-\lambda(\overline{\mathbf{R}} \cdot \mathbf{z}-\bar{R})-\mu\left(\mathbf{1}^{\top} \cdot \mathbf{z}-1\right)-\sum_{i=1}^{k} \alpha_{i}\left(p_{i}-\theta\right)
$$

wherein $\lambda \in \mathbb{R}, \mu \in \mathbb{R}, \alpha_{i} \in \mathbb{R}$ for $i=1, \ldots, k$ are the lagrange multipliers associated with the constraints.

Theorem 1 The first order condition is sufficient since we minimize a quadratic function under linear constraints (the Karush-Kuhn-Tucker conditions). It is given by

$$
\frac{\partial £\left(\mathbf{z}, \lambda, \mu ; \alpha_{i}, \ldots, \alpha_{k}\right)}{\partial \mathbf{z}}=\widetilde{\sigma} . \mathbf{z}-\lambda \overline{\mathbf{R}}^{\top}-\mu . \mathbf{1}=0
$$

Remark 1 The condition (10) is identical to the first order condition of the lagrangian function in the basic Markowitz problem (1959). With the introduction of a constraint on $p_{i}$ as in the problem (8), it is important to note that we reduce the number of assets in which we invest through a sub-portfolio with the same level of optimality that the initial portfolio.

Proposition 1 Considering the condition (10), the following of the resolution of the problem for the sub-portfolio defined from $p_{i}$ becomes the same as the initial Markowitz problem $(1952,1959)$ and, consequently the variance of the optimal portfolio is given by expression:

$$
\widetilde{\sigma}_{o p t}^{2}=\frac{C \widetilde{R}^{2}-2 A \widetilde{R}+B}{D}
$$


wherein

$$
\begin{aligned}
& A=\overline{\mathbf{R}} \widetilde{\sigma}^{-1} \mathbf{1}=\mathbf{1}^{\top} \widetilde{\sigma}^{-1} \overline{\mathbf{R}}^{\top} \\
& B=\overline{\mathbf{R}} \widetilde{\sigma}^{-1} \overline{\mathbf{R}} \\
& C=\mathbf{1}^{\top} \widetilde{\sigma}^{-1} \mathbf{1}
\end{aligned}
$$

The shape of the optimal sub-portfolios frontier is a parabola branch, whose base is called the minimum variance sub-portfolio.

\section{Numerical Experiments}

We show in this part, the choice test of the sub-portfolios optimal composition and the optimal frontier in four main comparative cases where the sub-portfolios will be dynamically compiled from the constraint on the PER $\left(p_{i}\right)$ of the shares which constitute these sub-portfolios (BASU, 1977). Particularly we suppose that investors don't put into their sub-portfolio risk-free asset, they will compose them on the basis of shares in the market composed at most with $n=19$ the titles in the first (except PALM CI belonging to the second one) compartment of the stock exchange listed in the Top $20 p_{i}$ officially published on the BRVM's website in January in connection with the performance achieved in the fourth quarter 2014. We emphasize that the fact of being part of the first compartment shows more or less a guarantee in the reliability of published results because of the requirements to be part of this compartment.

\subsection{Data Presentation}

We first present the classification of our 19 shares according to their $p_{i}$ officially published on behalf of the fourth quarter 2014. We emphasize that the ties were classified according to the decimal.

Table 1. Top 19 of the $p_{i}$ in the first compartment of the Regional Stock Exchange of Securities in West Africa on December 31, 2014

\begin{tabular}{clc}
\hline Rank & Assets & $p_{i}$ \\
\hline 1st & SAPH CI & 7 \\
2nd & BANK OF AFRICA BF & 9 \\
3th & SOGB CI & 12 \\
4th & PALM CI & 12 \\
5th & SONATEL SN & 12 \\
6th & BERNABE CI & 12 \\
7th & ONATEL BF & 12 \\
8th & ECOBANK TRANS. INCORP. TG & 12 \\
9th & BANK OF AFRICA CI & 13 \\
10th & TRACTAFRIC MOTORS CI & 13 \\
11th & BICI CI & 14 \\
12th & SICABLE CI & 15 \\
13th & BANK OF AFRICA BN & 15 \\
14th & AIR LIQUIDE CI & 16 \\
15th & TOTAL CI & 17 \\
16th & SITAB CI & 18 \\
17th & SOLIBRA CI & 19 \\
18th & BANK OF AFRICA NG & 21 \\
19th & SGB CI & 23 \\
\hline
\end{tabular}

Over the period from January 12 to February 6, 2015, that is to say four weeks of market, we collected datas about the assets named in table 1 from daily reports of the stock market (BOC). Those datas allowed us to get an estimation of the covariance matrix $\widetilde{\sigma}$ and the mean return vector $\overline{\mathbf{R}}$ of the 19 risked assets given on the following page. We specify that we do not take in account serial correlation because of the relatively very short multivariate time series we use for the estimation of $\widetilde{\sigma}$. 


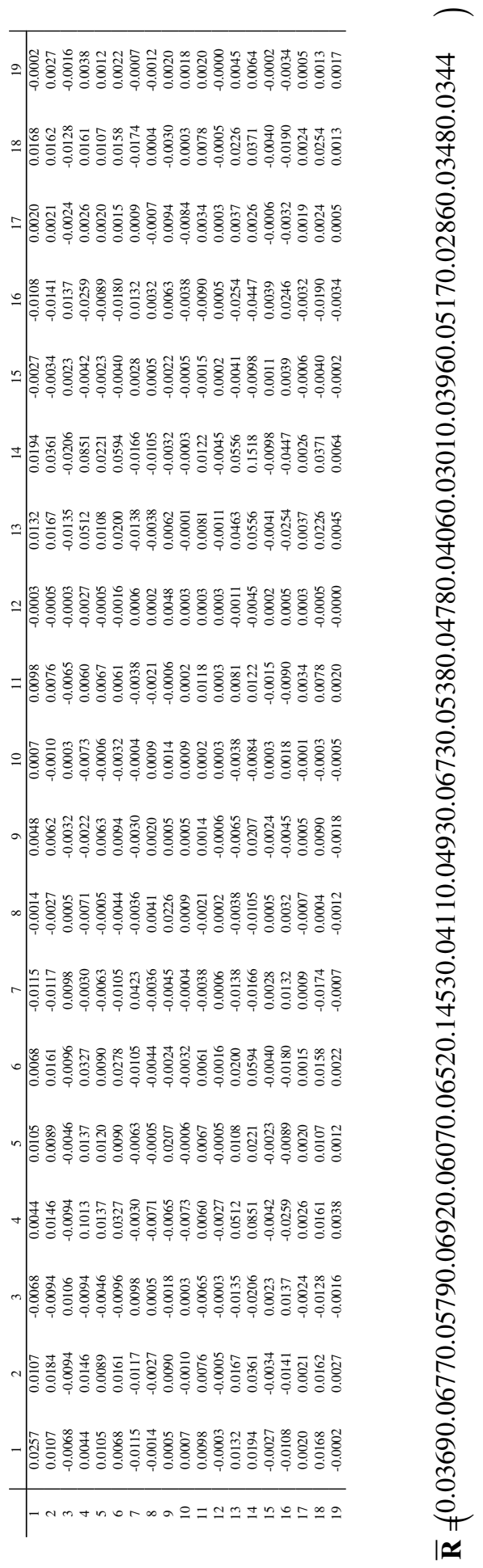




\subsection{Optimal Portfolios Frontier for $p_{i} \leq \theta$ with $\theta \in\{23,21\}$}

For $\theta=23$ years, the portfolios are composed from $k=19$ shares, ie the whole shares of our reduiced market and for $\theta=21$ years, $k$ starts to decrease, the number of shares which going to compose the sub-portfolio becomes 18 . We have a first comparison on figure 1 .

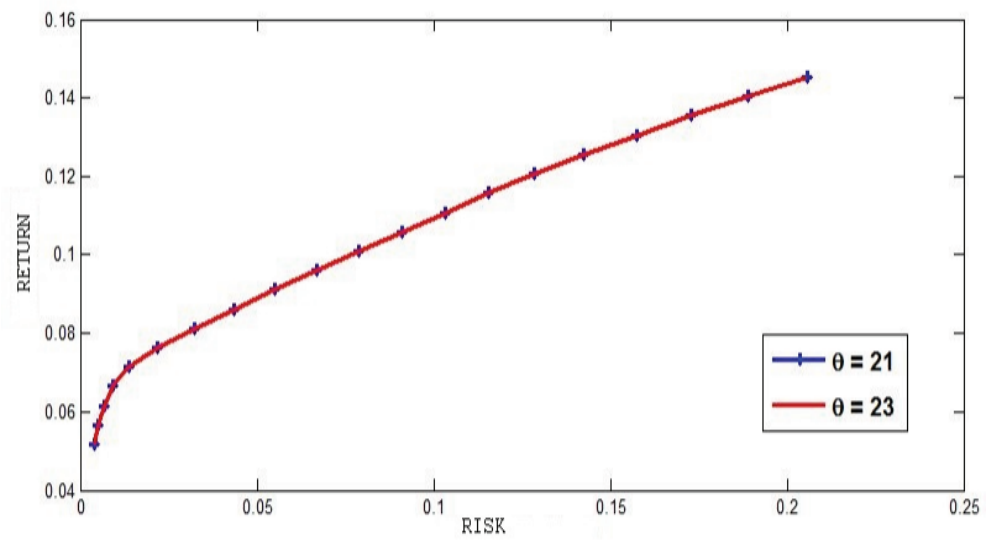

Figure 1. Optimal portfolios frontier for $\theta \in\{23,21\}$

\subsection{Optimal Portfolios Frontier for $p_{i} \leq \theta$ with $\theta \in\{21,19,18,17,16\}$}

We examine comparatively the cases where $\theta$ successively takes the values $21,19,18,17$ and 16 years, which corresponds to a decreasing of $\mathrm{k}$ with the respective values of 18, 17, 16, 15 et 14 shares. The result shows an equivalence of efficient frontiers presented in figure 2 .

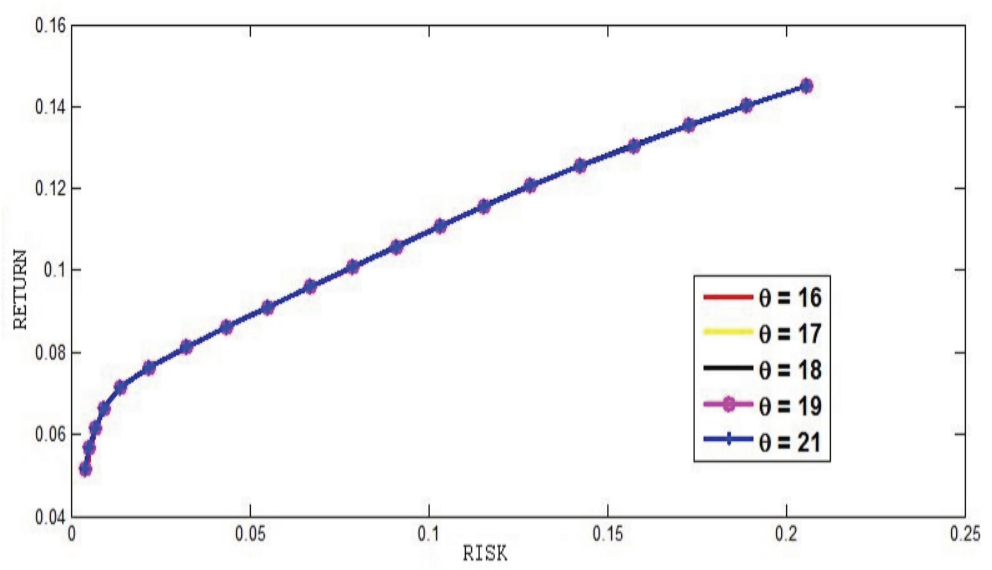

Figure 2. Optimal portfolios frontier for $\theta \in\{21,19,18,17,16\}$

4.4 Optimal Portfolios Frontier for $p_{i} \leq \theta$ with $\theta \in\{16,15,14,12\}$

The optimal frontier for $\theta=16$ becomes the reference curve of our comparison since we have that perfect convergence of optimal frontiers for $\theta>16$. The plotting of the frontiers for $\theta \in\{15,14,13,12\}$ shows convergence with the frontier for $\theta=16$ starting each one from a given point (cf Figure 3). We note three points, $C_{0}$ beginning of the convergence between $\theta=16$ and $\theta=15, X_{0}$ the this between $\theta=16$ and $\theta=13$ and $Y_{0}$ the beginning between $\theta=16$ and $\theta=12$. 


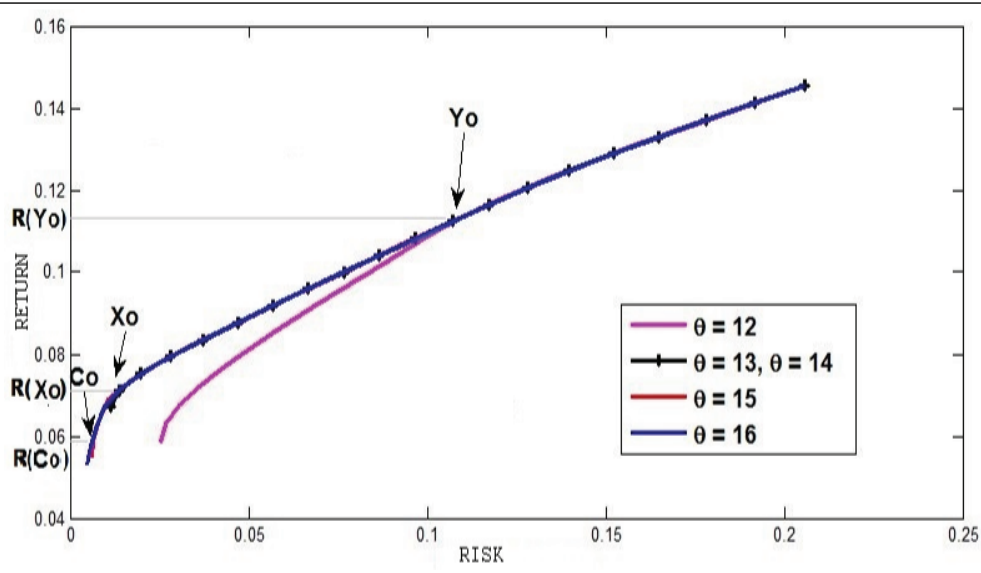

Figure 3. Optimal portfolios frontier for $\theta \in\{16,15,13,12\}$

When frontiers $\theta=14$ and $\theta=13$ are equivalent where $X_{0}$ is the beginning of the convergence with $\theta=16$ for both values. Thus it will be better to take $\theta=13$.

4.5 Optimal Portfolios Frontier for $p_{i} \leq \theta, \theta<12$

We notice a convergence loss for $\theta$, an integer inferior to 12, as we can see in Figure 4 with the comparison of $\theta=16$ and $\theta=12$.

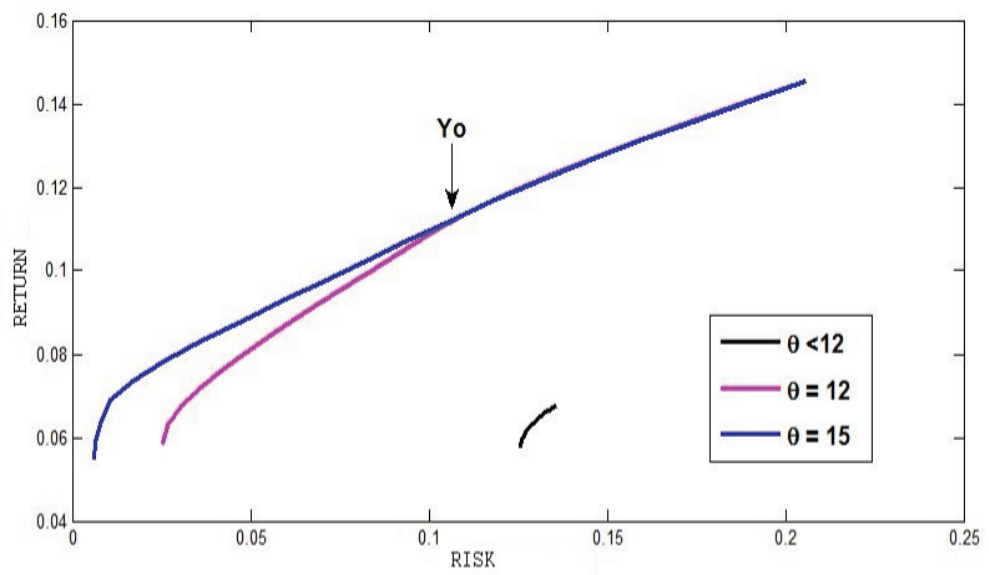

Figure 4. Optimal portfolios frontier for $\theta<12$

\section{Empirical Results}

The first simulations through figures 1 and 2 show us a perfect convergence of the sub-portfolio optimal frontiers for $\theta \in\{23,21,19,18,17,16\}$.

The approach by selected sub-portfolios on the basis of a constraint on the PER $p_{i}$ of 19 shares in the Regional Stock Market Securities, allows us to deduce as a first conclusion that an investor may restrict $p_{i}$ to 16 , and with this sub-portfolio of $k=14$, he can get exactly the same optimal portfolios than in focusing on $n=19$ shares or even all shares of the Regional Stock Market Securities.

Therefore, the equation that we will formulate is:

$$
P(\widetilde{R}):\left\{\begin{array}{l}
\min _{\mathbf{z}} \frac{1}{2} \mathbf{z}^{\top} \cdot \widetilde{\sigma} \cdot \mathbf{z} \\
s / c: \\
p_{i} \leq 16 \\
\overline{\mathbf{R}} \cdot \mathbf{z}=\widetilde{R} \\
\mathbf{z}^{\top} \cdot \mathbf{1}=1
\end{array} \quad i \leq n\right.
$$

Thus, we consider the optimal frontier for $\theta=16$ as a reference curve and we decrease again successively to 15 , 14 et 12 . The point $C_{0}$ although being the beginning of the convergence between the frontiers of the sub-portfolios 
$\theta=16$ et $\theta=15$ do not illustrate clearly the difference of these two frontiers. That is solve the problem (13) almost amounts to solve it for $\theta=15$ :

$$
P(\widetilde{R}):\left\{\begin{array}{l}
\min _{\mathbf{z}} \frac{1}{2} \mathbf{z}^{\top} \cdot \widetilde{\sigma} \cdot \mathbf{z} \\
s / c: \\
p_{i} \leq 15 \\
\overline{\mathbf{R}} \cdot \mathbf{z}=\widetilde{R} \\
\mathbf{z}^{\top} \cdot \mathbf{1}=1
\end{array} \quad i \leq n\right.
$$

The results that we are going to emphasize at this level will be lied in the points $X_{0}$ and $Y_{0}$ throughout their respective yields $R\left(X_{0}\right) \simeq 0,075$ or $7,5 \%$ and $R\left(Y_{0}\right) \simeq 0,115$ or $11,5 \%$ (cf figure 3 ).

Then we may express the three following conclusions:

1. The investor who aims an objective return $\widetilde{R}<R\left(X_{0}\right)$ will solve the problem (14) for $k=13$ shares.

2. The investor whose aim is an objective return $\widetilde{R} \in] R\left(X_{0}\right), R\left(Y_{0}\right)$ [, must focus on the optimization of a subportfolio composed by shares which Price Earnings Ratio $p_{i}$ is at most equal to 13 hence the resolution of an optimization problem for $\theta=13$ with $k=10$ shares.

3. If he sets a target of $\widetilde{R}>R\left(Y_{0}\right)$, he must solve at the beginning the optimization problem for $\theta=12$ by focusing on the research of the composition $z$ for a sub-portfolio of $k=8$ shares instead of a bigger portfolio of $n=19$ shares while knowing that the optimal frontier will be the same.

\section{Discussion}

This paper is a contribution to the development effort of the west african financial market, the Regional Stock Market Securities and was the opportunity for us to study selected sub-portfolios optimization on the base of Price Earnings Ratio of shares published at the end of the last quarter of the year 2014. Indeed, we have followed from January 12 to February 06 the returns of 19 companies of first section that appear in the ranking of the 20 best Price Earnings Ratio of the last quarter.

The resolution of the optimization problem of Markowitz with a dynamic constraint on the Price Earnings Ratio, which change this problem to an optimal dynamic sub-portfolio selection, allows us to see that being focused on a Price Earnings Ratio restriction we can optimize the number of shares which compose the portfolio before targeting a given global output. In the case of the Regional Stock Market Securities and according to the datas with which we have worked, we might determine depending on the objective return interval, the number of years during which it is optimal to restrict the Price Earnings Ratio, as well as the minimal base of shares on which we have to focus in order to reach this objective return.

We long to continue this work through a multiobjective optimization, which includes the research about the Price Earnings Ratio impact, and uses real values, without formulating a hypothesis about the outputs distribution. At the same time, we are going to focus on the risk measure and especially we will try to collect datas for a more long time. However, this is a real difficulty when we notice the flaws of our stock exchange system in this field.

\section{Acknowledgements}

This work was supported by NLAGA-Simons project. We do thank as well Dr. Moustapha Sene of Gaston Berger University in Senegal for all his help during the research.

\section{References}

Basu, S. (1977). Investment performance of common stocks in relation to their Price-Earnings Ratios: A test of the efficient market hypothesis. The Journal of Finance, 32(3), 663-682.

Beaver, W., \& Morse, D. (1978). What Determines Price-Earnings Ratios?. Financial Analysts Journal, 34(4), 65-76. http://dx.doi.org/10.2469/faj.v34.n4.65

Beder, T. (1995). VaR : seductive but dangerous. Financial Analysts Journal, 51, 12-24. http://dx.doi.org/10.2469/faj.v51.n5.1932

Ciarlet, P. (1998). Introduction l'analyse numrique matricielle et l'optimisation, Dunod.

Cornuejols, G., \& Tutuncu, R. (2007). Optimization methods in finance, Cambridge University Press. 
Debreu, G. (1952). Definite and semidefinte quadratic forms. Econometrica, 20, 295-300. http://dx.doi.org/10.2307/1907852

El hachloufi, M., Guennoun, Z., \& Hamza, F. (2012). Stocks Portfolio Optimization Using classification and Genetic Algorithms. Applied Mathematical Sciences, 6(94), 4673-4684.

Fieldsend, J, Mitatko, J., \& Peng, M. (2004). Cardinality constrained portfolio optimization, 5th International Conference on Intelligent Data Engineering and Automated Learning (IDEAL 2004), Exeter, England, Lecture Notes in Computer Science, vol 3177, p.788-793.

Gilli, M, Kllezi, E. (2000, July 6-8). Heuristic Approaches for Portfolio Optimization. In: Sixth International Conference on Computing in Economics and Finance of the Society for Computational Economics. Barcelona.

Hamza, F., \& Janssen, J. (1995). Portfolio Optimization Model Using Asymmetric risk Functions. Actuarial Approach for Financial Risks, 3 Bis, 3-32.

Jorion, P. (2000). Value at Risk, The New Benchmark for Managing Financial Risk, 2nd Edition McGraw-Hill.

Jobst, N, Horniman, M, Lucas, C., \& Mitra, G. (2001). Computational aspects of alternative portfolio selection models in the presence of discrete asset choice constraints. Quantitative Finance, 1, 1-13. http://dx.doi.org/10.1088/1469-7688/1/5/301

Konno., \& Yamazaki. (1991). A Mean Aboluate Deviation Portfolio Optimisation Model and Application to Tokyo Stock Market. Management Science, 37, 519-531. http://dx.doi.org/10.1287/mnsc.37.5.519

Makoto, O. (2011). Optimisation of Finite Dimentional Structures, Hiroshima University, Higashi-Hiroshima, Japan.

Markowitz, H. M. (1952). Portofolio Selection. Journal of Finance, 7(1), 77-91.

Markowitz Harry, M. (1959, 1970, 1991). Portofolio Selection: Efficient Diversification of Investments, Wiley, 1959, Yale University Press, 1970, Basil Blackwell, 1991.

Penman, S. H. (1996). The Articulation of Price-Earnings Ratios and Market-to-Book and the evaluation of Growth. Journal of Accounting and Research, 34(2), 235 (abstract). http://dx.doi.org/10.2307/2491501

Rudd., \& Rosenbeg. (1979). Realistic Portfolio Optimisation. TIMS Studies in the Management Science, 11, $21-46$.

Schaerf, A. (2002). Local Search Techniques for Constrained Portfolio Selection Problems. Computational Economics, 20, 177-190. http://dx.doi.org/10.1023/A:1020920706534

Sharpe. (1967). A Linear programming Algorithm for Mutual fund Portfolio Selection. Management Science, 13, 499-510.

Sharpe. (1971). A Linear Programming Approximation for General Portfolio Selection Problem. J.Quantitative Anal, 6, 1263-1275.

Speranza. (1993). Linear Programming Models for Portfolio Optimization. Finance, 14, 107-123.

Stone. (1973). A Linear Programming Formulation of the General Portfolio Selection Problem. J.Quantitative Anal, 6, 107-123.

Yoshimoto. (1996). The Means Approch to Portfolio Optimisation Subject to Transaction Costs. Journal of the Operation Research, Society of Japan, pp.99-117.

Zenios., \& Pang. (1993). A Mean Aboluate Deviation Portfolio Optimisation for Mortgage Backed Securities. Annals of Operations Research, 45(9), 433-450.

\section{Copyrights}

Copyright for this article is retained by the author(s), with first publication rights granted to the journal.

This is an open-access article distributed under the terms and conditions of the Creative Commons Attribution license (http://creativecommons.org/licenses/by/3.0/). 